\title{
Sequence analysis of loci Rpv10 and Rpv3 for resistance against grapevine downy mildew (Plasmopara viticola)
}

\author{
Jens Dudenhöffer ${ }^{1}$, Florian Schwander ${ }^{1}$, Reinhard Töpfer ${ }^{1}$, Eva Zyprian ${ }^{1}$ \\ ${ }^{1}$ Julius Kühn-Institut, Institute for Grapevine Breeding Geilweilerhof, Siebeldingen \\ Email of corresponding author: eva.zyprian@jki.bund.de
}

Among several other partners the Julius Kühn-Institut at Siebeldingen is involved in a cooperative project called "Bacchus" funded by the the interregional program "INTERREG IV Upper Rhine" of the objective "European territorial cooperation". The grant is provided by the "European Regional Development Fund" (ERDF) allocated by the European Union (EU).

The oomycete Plasmopara viticola causing downy mildew is one of the most important pathogens of grapevine (Vitis vinifera L.). Especially all traditional European cultivars used for wine production are highly susceptible. As a result an extensive amount of fungicide applications are necessary to enable the cultivation of healthy grapes. For that reason newly bred mildew resistant varieties are a major contribution to reduce the amount of fungicides for sustainable viticulture.

Many North American Vitis species possess resistance genes due to coevolution with Plasmopara viticola. Beyond that Asian Vitis species also exhibit such resistances although their evolutionary origin is not understood yet. Among others, the two loci Rpv3 (American origin e. g. cv. 'Regent') and Rpv10 (Asian origin e. g. cv. 'Solaris') are known.

Up to now we could assemble most of the region of $R p v 10$ via amplicon sequencing. Rpv3-linked molecular SSR-markers were used to screen for contigs from the Rpv3 locus in two different Next Generation Sequencing datasets of resistant cultivars. Three possible candidate genes for each downy mildew resistance locus could be found so far. They possess protein domains showing similarity to resistance genes found in other plant species. In comparison to the grapevine reference genome of 'Pinot noir' (PN40024) - which is susceptible for Plasmopara viticola - some genes show major differences in essential protein domains.

RNASeq analysis was done of plants carrying Rpv10, Rpv3 or none of these loci. The samples were collected in the very early hours after inoculation with the pathogen to investigate the expression level of the genes possibly conferring resistance against Plasmopara viticola. The analysis of the RNASeq data is in progress. 